\title{
O campo da Saúde Coletiva na perspectiva das disciplinas
}

\author{
The field of Collective $\mathrm{H}$ ealth in the courses perspective
}

Everardo DuarteN unes $^{1}$

LiraneElize Ferreto ${ }^{1}$

Ana Luiza de Oliveira e Oliveira ${ }^{1}$

Juliana Luporini do Nascimento ${ }^{1}$

$\mathrm{Nelson}$ Filice deBarros ${ }^{1}$

M arcelo Eduardo Pfeiffer Castellanos ${ }^{2}$

\footnotetext{
${ }^{1}$ Departamento deM edicina Preventiva e Social, FaculdadedeCiências M édicas, Universidade Estadual deCampinas. Cidade Universitária Zeferino Vaz, Distrito Barão Geraldo. 13083-970 Campinas SP. evernunes@uol.com.br ${ }^{2}$ Instituto deSaúde Coletiva, Universidade Federal da Bahia.
}

Abstract This articlediscusses the field of Collective $\mathrm{H}$ ealth from the disciplines that structure the curriculum of the Collective Health area postgraduate courses in 2006. From the database of programs, including master's, doctoral and professional masters were classified 1,171 subjects, in what we call disciplinary fields, linking this to the P. Bourdieu's notion of champ (field). Twenty-eight disciplinary fields were described, in three large groups. It was concluded that the field of collective health has a wide range of disciplinary fields, especially the frequency: Epidemiology (16\%) , H umanities and Social Sciences in H ealth (7.9\%); Curriculum (7.9\%); Systems and Health Services $(7.4 \%)$, Environment and $H$ ealth (7.2\%) Health Policies (6.3\%).

Key words Collective health, Discipline, Disciplinary field
Resumo Este artigo aborda o campo da Saúde Coletiva a partir das disciplinas que estruturam a grade curricular dos cursos de pós-graduação da área da Saúde Coletiva, no ano de 2006. A partir do banco de dados dos programas, que incluem mestrado, doutorado emestrado profissionalizante foram classificadas 1.171 disciplinas, no que denominamos de campos disciplinares, associando esta nomenclatura à noção de campo procedente das abordagens de Bourdieu. São descritos 28 campos disciplinares em três grandes blocos. Concluise que o campo da Saúde Coletiva apresenta uma grandediversidade de campos disciplinares, destacando-se pela frequência: Epidemiologia (16\%); Ciências H umanas e Sociais em Saúde (7,9\%); Didática $(7,9 \%)$; Sistemas e Serviços de Saúde (7,4\%); A mbiente e Saúde (7,2\%); Políticas de Saúde(6,3\%).

Palavras-chave Saúdecoletiva, Disciplinas, Campo disciplinar 
Introdução

0 campo da SaúdeColetiva éum dos mais férteise avançados atual mente na árvore dos saberes di sciplinares ${ }^{1}$.

Estudar um campo de saberes e práticas é enfrentar o caráter transformador que ele apresenta em sua trajetória. Isto que está presente, como processo histórico, em todos os campos do conhecimento e na tradição epistemológica francesa com Bachelard ${ }^{2}$, Canguilhem ${ }^{3}$ e Foucault $^{4}$, e que se associa à vertente sociológica de Bourdieu 5 , estende-se à área da Saúde Coletiva de forma exemplar.

$N$ ão é estranho que muitos que se dedicaram à tarefa de compreender (verstehen) a saúde coletiva situam claramente a diferença entre área e campo. Particularmente, nessa direção, Luz ${ }^{1}$ sintetiza de forma objetiva que A dotando como guia analítico a categoria de campo torna-se menos difícil a compreensão da coexistência e multiplicação dos saberes e práticas na saúde coletiva. Esta categoria nos permite ver como um domínio específico de saberes e práticas no campo das ciências distribui hierarquicamente seus discursos e os atores/agentes que os emitem, num conjunto semi estruturado em processo, em que a disputa, muitas vezes conflituosa, pela hegemonia do poder simbólico está sempre presente.

Prossegue a autora, anotando: Evidentemente, esta distribuição não se descola da cultura e da estrutura social, das forças sociais quea compõem, em um momento espećífico de sua construção histórica, bem como dos imperativos de ação ("necessidades") quetal momento coloca para a sociedade como um todo, para suas instituições, seus saberes e suas práticas normativas.

Sem dúvida, a noção de campo, muito mais flexível que a de estrutura, encontra ampla aceitação nos estudos da saúde coletiva ${ }^{1,6-8}$ e tornase um precioso instrumento conceitual. Textualmente, o campo científico éum campo social como outro qualquer, com suas relações de força emonopólios, suas lutas e estratégias, seus interesses e lucros, mas onde todas essas invariantes revestem formas específicas ${ }^{5}$. Para Bourdieu, o campo científico étambém um espaço deluta concorrencial, onde o que está em jogo especificamente nessa luta é o monopólio da autoridade científica definida, de maneira inseparável, como capacidade técnica e poder social; ou, se quisermos, o monopólio da competência científica, compreendida enquanto capacidade defalar eagir legitimamente (isto é, de maneira autorizada e com autoridade), queé socialmente outorgada a um agente determinado ${ }^{5}$.
Sem tirar o mérito da complexidade presente em todos os campos do conhecimento, e Piaget $^{9}$ mostrou muito bem que não há campo exclusivamente disciplinar, pode-se dizer que o da saúde tem essa característica ampliada. E isto está presente em todas as definições da saúde coletiva, como sendo inter, multi e transdisciplinar. Embora esta seja uma noção compartilhada pelos autores deste trabalho, o ponto de partida não foi situar a saúde coletiva dedutivamente, ou seja, a partir de uma definição prévia do campo, mas reconstruí-la a partir dos seus segmentos disciplinares, indutivamente. Assim, a partir desta perspectiva metodológica - das partes para o todo - busca-se uma cartografia do campo visualizado a partir das disciplinas nominadas nos cursos e classificadas em campos disciplinares, agregando diversas disciplinas pelas semelhanças, que conformam os subcampos do campo maior da saúde coletiva.

\section{M etodologia}

A principal fonte de dados foi a relação de disciplinas (obrigatórias e eletivas) de cursos de pósgraduação em Saúde Coletiva de 2006, disponíveis nos registros da Coordenação de A perfeiçoamento dePessoal de Nível Superior (CAPES). A relação completa das disciplinas pelas instituições é apresentada na Tabela 1.

\section{Resultados}

Foram classificadas 1.171 disciplinas, independente de serem de mestrado, doutorado ou mestrado profissionalizante. Os dados existentes não permitiram saber se são disciplinas obrigatórias ou eletivas.

Agrupadas em grandes subáreas do conhecimento, aqui denominadas de campos disciplinares, verifica-se pela Tabela 1 e Gráfico 1 que a Epidemiologia apresenta o maior número dedisciplinas, totalizando 16\%; as Ciências H umanas eSociais em Saúde apresentam 7,9\%; a Didática, 7,9\%; os Sistemas e Serviços de Saúde, 7,4\%; AmbienteeSaúde, 7,2\%; Políticas deSaúde, 6,3\%. Além desses seis grupos de campos disciplinares, que apresentam as maiores porcentagens de frequência, foram catalogados mais 23 grupos disciplinares, também considerados como subáreas. Como pode ser visto, não foi feita distinção entre a nomeação de disciplinas convencionais e outras englobadas como Didática, O ficinas, Ban- 
Tabela 1. Distribuição dos campos disciplinares dos cursos de pós-graduação em saúde coletiva por número e porcentagem de disciplinas.

\begin{tabular}{lrr}
\hline \multicolumn{1}{c}{ Campos disciplinares } & N $~$ & \multicolumn{1}{c}{$\%$} \\
\hline Epidemiologia & 188 & $16 \%$ \\
Didática & 93 & $8 \%$ \\
Ciências Humanas e Sociais em Saúde & 93 & $8 \%$ \\
Sistemas e Serviços de Saúde & 87 & $7,43 \%$ \\
Ambiente e Saúde & 84 & $7,17 \%$ \\
Políticas de Saúde & 74 & $6,32 \%$ \\
Diversos & 60 & $5,12 \%$ \\
M etodologia de Pesquisa & 54 & $4,61 \%$ \\
Saúde do Trabalhador & 46 & $3,93 \%$ \\
Bioestatística & 41 & $3,50 \%$ \\
Saúde da Mulher, Criança e Adolescente & 34 & $2,90 \%$ \\
Estatística & 34 & $2,90 \%$ \\
Teses e Dissertações & 28 & $2,34 \%$ \\
Saúde Coletiva e Saúde Pública & 28 & $2,34 \%$ \\
Pesquisa Qualitativa & 23 & $1,96 \%$ \\
Nutrição & 22 & $1,88 \%$ \\
Informação em Saúde & 20 & $1,70 \%$ \\
Informática & 18 & $1,54 \%$ \\
Ciência e Tecnologia & 18 & $1,54 \%$ \\
Pesquisas Interdisciplinares & 17 & $1,45 \%$ \\
Economia da Saúde & 17 & $1,45 \%$ \\
Oficinas & 16 & $1,37 \%$ \\
Ética e Bioética & 16 & $1,37 \%$ \\
Educação & 15 & $1,29 \%$ \\
Banco de Dados & 14 & $1,19 \%$ \\
Saúde Bucal & 10 & $0,85 \%$ \\
Saúde M ental & 9 & $0,77 \%$ \\
Geografia da Saúde & 8 & $0,68 \%$ \\
M edicamentos & 4 & $0,34 \%$ \\
Total & 1.171 & $100 \%$ \\
\hline & & \\
\hline & & \\
& &
\end{tabular}

co de Dados, Teses e Dissertações, Pesquisas Interdisciplinares.

Pelos dados obtidos, há, além da maior concentração de disciplinas do primeiro grupo, um segundo grupo com algum destaque. Dentre essas disciplinas, M etodologia de Pesquisa tem $4,6 \%$ do total de disciplinas e, em ordem decrescente, Saúde do Trabalhador, 3,9\%; Bioestatística, 3,5\%; Estatística, 3,0\%. Um terceiro grupo, o maior dos três, com porcentagens quevariam de 0,3\% (M edicamento) a 2,9\% (Saúde da Mulher, Criança e Adolescente), há outros dezoito campos disciplinares. Verifica-se que esses campos disciplinares englobam uma grande diversidade: Geografia da Saúde( $0,7 \%)$; Saúde M ental (0,8\%); Saúde Bucal (0,9); Banco de Dados (1,2\%); Edu-

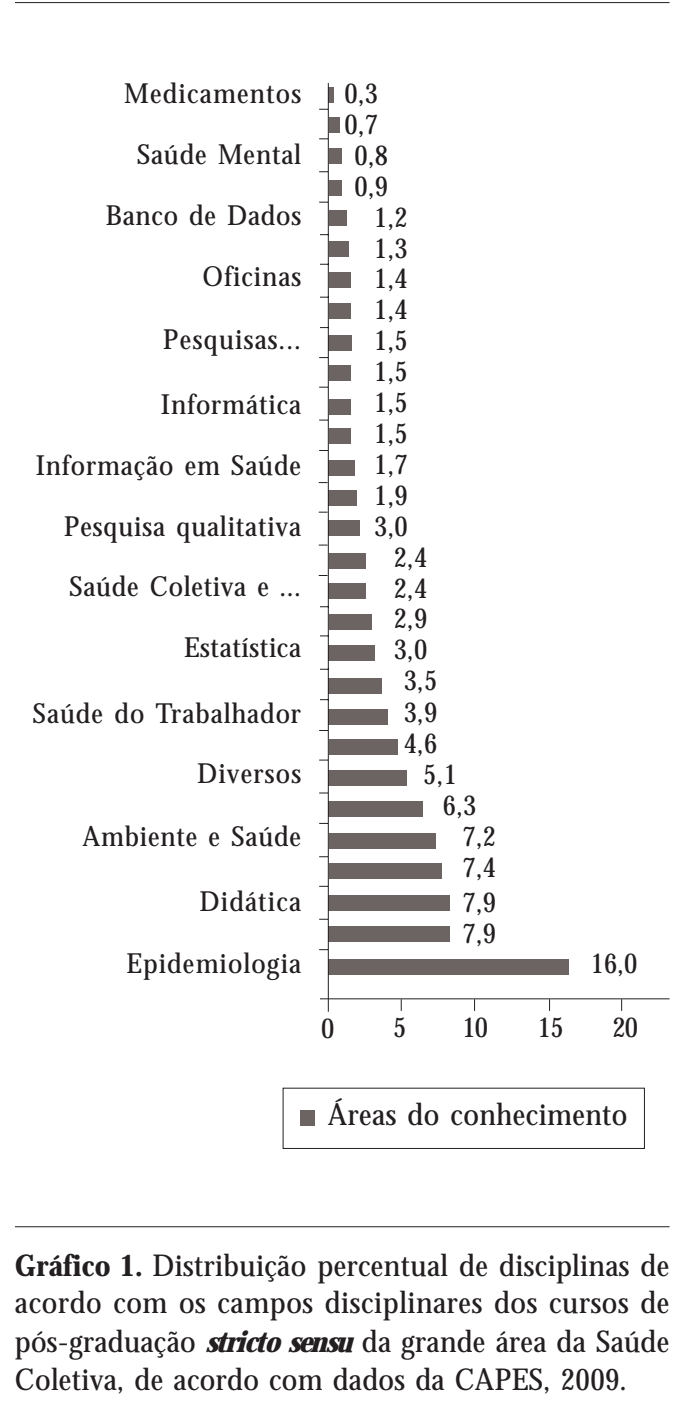

cação (1,3\%); Oficinas (1,4\%); Ética e Bioética $(1,4 \%)$; Pesquisas Interdisciplinares (1,5\%); Economia da Saúde (1,5\%); Informática (1,5\%); Ciência eTecnologia (1,5\%); Informação em Saúde $(1,7 \%)$; Nutrição (1,9\%); Pesquisa Qualitativa (2,0\%); Teses e Dissertações (2,4\%) e Saúde Coletiva eSaúde Pública (2,4\%).

\section{Discussão}

Os dados permitem uma primeira aproximação com a noção que foi tomada como orientadora desta análise- a de campo. Em realidade, constata-se uma grande diversidade de áreas de conhecimento (disciplinas), mas o campo da Saúde 
Coletiva pode ser nucleado hierarquicamente, pela presença quantitativa, em cinco grandes "campos disciplinares": a Epidemiologia, as Ciências Humanas e Sociais, os Sistemas e Serviços de Saúde, Ambiente e Saúde e Políticas de Saúde. Comparando, mas lembrando que as pesquisas adotaram metodologias distintas, com os dados da primeira avaliação da estrutura curricular, verifica-sequea epidemiologia continua a liderar quantitativamente a área. Na primeira avaliação, foram analisadas 38 áreas de concentração: no mestrado, na grande área das Ciências da Saúde, a Epidemiologia apresentava 40,9\% dos conteúdos de ensino; Planejamento eAdministração em Saúde, 27,3\%, Bioestatística, 23,6\%; na grande área das Ciências Humanas, os conteúdos englobados na rubrica Filosofia (incluindo, dentre outras, Metodologia, Sociologia, Ciência Política, História e Educação) atingiram 42,8\%. No doutorado, na grande área das Ciências da Saúde e a das Ciências H umanas, os valores encontrados foram iguais ( $45 \%$ ) e, no interior dessas áreas de conhecimento, a distribuição dos principais conteúdos é bastante semelhante ao mestrado: epidemiologia ebioestatística, planejamento eadministração em saúde e metodologia e epistemologia. Observou-se, naquele momento, a presença reduzida das disciplinas incluídas na grande área das Ciências Biomédicas, Engenharia e Ciências Sociais A plicadas ${ }^{10}$.

Interessante observar que, na pesquisa atual, al guns campos disciplinares, como os relacionadosà Ambientee Saúdee Políticas de Saúde, figuravam na pesquisa anterior, mas assumem destaque na atualidade. De outro lado, aparecem Geografia da Saúde, Ética e Bioética, Economia da Saúde, em pequenas proporções, mas que não podem deixar de ser citados.

Quando da avaliação anterior, M inayo ${ }^{11}$ lembrava que uma questão não resolvida referia-se a um núcleo central de disciplinas que ao contrário dosquedefendem uma ampla autonomia, essegrupo entende quea demarcação de um conhecimento básico preserva a identidade da área e permite liberdade de exploração de objetos fronteiriços, também essenciais para a teoria e a prática da Saúde Coletiva. Discutiu-se, também, que havia necessidade "de se proceder a um esforço para aprofundar os modelos teóricos e conceituais do campo, a fim de se proporem conteúdos indispensáveise não necessariamente sob a forma de disciplinas".

Retomamos essas questões e mesmo sem poder aprofundar os conteúdos disciplinares, verificamosqueas sugestões da avaliação de 1997 propunham que Epidemiologia, Bioestatística,
Saúde Ambiental, Comportamento/Educação e Sistemas e Serviços de Saúde, às quais se acrescentaram H istória e Filosofia da Saúde Pública, Metodologias de Pesquisa, com exceção de Comportamento/Educação, não são estranhas aos cursos atuais.

Como apresentamos os dados deforma agregada e classificatória, é necessário que se destaque a enorme diversidade temática que está no interior do que denominamos campos disciplinares. Em realidade, essa ideia apresenta uma especial proximidade com a noção de "campo científico" na abordagem de Bourdieu evem sendo amplamente utilizada nas análises das transformações históricas de temas que, ao longo do tempo, sistematizaram conhecimentos e saberes com especificidades teóricas e metodológicas. Dentre os inúmeros exemplos, podem ser citados: história econômica ${ }^{12}$, patrimônio históri$\mathrm{CO}^{13}$, estudos feministas ${ }^{14}$, prática deensino ${ }^{15}$, etc.

No caso da Saúde Coletiva, como já observamos, são 29 campos disciplinares cujos conteúdos temáticos variam em relação às diversas possibilidades de análises: macro ( políticas de saúde, estado, globalização e reestruturação produtiva, organização de programas e serviços de saúde, desigualdadeem saúde, desenvolvimento de políticas de saúde, etc.); micro (indivíduo e sociedade); conceituais (elementos teóricos de saúde e sociedade, representações sociais, bases teóricas da promoção social, conceito de risco, etc.); qualitativos (estudos sobre subjetividade, métodos qualitativos); quantitativos (análises estatísticas); epidemiológicos (gerais - aspectos teóricos, metodológicos e específicos - diversas doenças, envelhecimento, atividadefísica, violência, etc.); históricos (história da prática médica, história da saúde pública, história da saúde col etiva); educacionais (educação ambiental, educação e sociedade, educação enutrição, etc.); ambientalistas (metodologias deanálises em saúdee ambiente, legislação, política e gestão, poluição e qualidade de águas, riscos químicos, biossegurança, toxicologia, vigilância, etc.); ciclo de vida (estudos sobre infância, adolescência, idadeadulta evelhice); gê nero (saúde da mulher, saúde reprodutiva); informacionais, comunicativos e tecnológicos (aspectos conceituais, gestão, políticas, estratégias, tendências); econômicos (análisedecustos, financiamento, mercado); metodológicos (estratégias e fundamento de investigação, relações ciência e método, etc.); interdisciplinaridade (etnoepidemiologia, genética e biomedicina, trabalho/educação/ ambiente/saúde); éticos (ética aplicada à saúde pública, ética na pesquisa, ensino); geográficos 
(metrópoles, riscos na habitação); referentes à nutrição (relações com doenças crônicas, consumo al imentar, segurança al imentar, etc. ); referentes à saúde mental ( relações com trabalho, saúde pública, etc.); trabalho (acidentes, riscos ocupacionais, doenças, fatores psicossociais, ergonomia, saúde do trabalhador, etc.); bioestatísticos (modelos de regressão, análise de sobrevivência, etc.); referentes à saúdebucal (diagnóstico bucal, odontologia social, planejamento, etc.); banco de dados (análise, gerência de dados, etc.). A parecem, também, dois grupos de disciplinas sob os títulos de Didática (aplicada à saúde, seminários, avaliação de ensino, etc.) e Teses e Dissertações.

Um ponto que acreditamos mereça ser comentado é que a maior ou menor participação dos campos disciplinares em relações de complementaridade depende da estrutura dos cursos. Assim, hoje, tornou-se mais comum que as áre as de concentração estabeleçam algum grau de relacionamento, ou seja, construam os programas curriculares tornando obrigatório que os alunos cursem pelo menos uma ou duas disciplinas obrigatórias fora da sua área de concentração. De outro lado, cumpre lembrar que o dimensionamento das disciplinas mantém estreita relação com o contexto institucional em quese estruturam os cursos de pós-graduação. Esse contexto pode ser o de uma escola ou faculdade, de um departamento emesmo o deum instituto. Como podemos verificar pela Tabela 2, as disciplinas se distribuem de acordo com o tamanho das instituições; assim, do total das 1.171 disciplinas, a maior concentração encontra-se na ENSP/Fiocruz, seguida pela USP (FM e FSP). A variação de número de disciplinas é grande, dependendo das áreas de concentração existentes.

Tabela 2. Distribuição de disciplinas por instituição de ensino.

\begin{tabular}{lc}
\hline \multicolumn{1}{c}{ Instituição de ensino } & $\mathrm{N}^{\circ}$ \\
\hline Centro de Pesquisa Aggeu Magalhães Fiocruz & disci plinas \\
Faculdade de Ciências M édicas da Santa Casa de São Paulo (FCM /SCM SP) & 48 \\
Fundação Oswaldo Cruz (Fiocruz) & 17 \\
Universidade Estadual do Ceará (UECE) & 307 \\
Universidade Estadual de Feira de Santana (UEFS) & 27 \\
Universidade Estadual de Londrina (UEL) & 26 \\
Universidade do Estado do Rio de Janeiro (UERJ) & 21 \\
Universidade Federal da Bahia (UFBA) & 28 \\
Universidade Federal do Ceará (UFC) & 47 \\
Universidade Federal do Espírito Santo (UFES) & 22 \\
Universidade Federal de M inas Gerais (UFM G) & 18 \\
Universidade Federal de Mato Grosso (UFMT) & 55 \\
Universidade Federal de Pernambuco (UFPE) & 16 \\
Universidade Federal de Pelotas (UFPEL) & 15 \\
Universidade Federal do Rio Grande do Sul (UFRGS) & 60 \\
Universidade Federal do Rio de Janeiro (UFRJ) & 39 \\
Universidade Federal de Santa Catarina (UFSC) & 24 \\
Universidade Luterana do Brasil (ULBRA) & 17 \\
Universidade Estácio de Sá (UN ESA) & 25 \\
Universidade Estadual Paulista Júlio de M esquita/ Botucatu (UNESP) & 18 \\
Universidade Estadual de Campinas (UNICAMP) & 27 \\
Universidade Federal de São Paulo (UNIFESP) & 21 \\
Universidade de Fortaleza (UNIFOR) & 23 \\
Universidade Católica de Santos ( UNISANTOS) & 22 \\
Universidade do Vale do Rio dos Sinos (UNISINOS) & 40 \\
Universidade de São Paulo/ Ribeirão Preto (USP/RP) & 15 \\
Universidade de São Paulo (USP) / Faculdade de M edicina e Faculdade de Saúde Pública & 17 \\
Total & 176 \\
\hline & 1.171 \\
\hline
\end{tabular}




\section{Considerações finais}

Deum modo geral, percebemos quea configuração do campo da Saúde Coletiva não sofreu alterações em sua estrutura na última década; há, sem dúvida, a ampliação de conteúdos nos campos disciplinares, mas sem grandes inovações. Como assinala $L Z^{1}$, revendo várias publicações de 1995 a 2005, o núcleo central da Saúde Coletiva em termos conceituais e práticos mantém aproximações, em termos paradigmáticos, com as ciências humanas, medicina, epidemiologia clássica, planejamento, gestão e avaliação das políticas de saúde, materializadas em programas e serviços. Como apontamos, há uma expressiva diversidade temática que se reflete quando os cursos intitulam suas disciplinas e dessa forma não se en quadram, muitas vezes, nos títulos convencionais, ou seja, particularizam conteúdos.

\section{Referências}

1. Luz MT. Complexidade do Campo da Saúde Coletiva:multidisciplinaridade, interdisciplinaridade, e transdisciplinaridade de saberes e práticas análise sócio-histórica de uma trajetória paradigmática. Saúde soc. 2009; 18(2):304-311.

2. Bachelard G. A formação do espírito científico. Rio de Janeiro: Contraponto; 1996.

3. Canguilhem G. O objeto das história das ciências. Tempo Brasileiro 1972; 28:7-21.

4. Foucault M. A arqueologia do saber. Petrópolis: Vozes; 1972.

5. Bourdieu P. O campo científico. In: Pierre Bourdieu: Sociologia. São Paulo: Ática; 1983.

6. Paim JS, Almeida Filho N. Saúde coletiva: uma "nova saúde pública" ou campo aberto a novos paradigmas. Rev. Saude Publica 1998; 32(4):299-316.

7. Nunes ED. Pós-graduação em saúde coletiva no Brasil: histórico e perspectivas. Physis 2005; 15(1):13-38.

8. Campos GWS. Saúde pública e saúde coletiva: campo e núcleo de saberes e práticas. Cien Saude Colet 2000; 5(2):219-230.

9. Piaget $J$. Los dos problemas principales de la epistemología de las ciencias del hombre. In: Piaget J, editor. Epistemología de las ciencias humanas. Buenos Aires: Proteo; 1972.

10. Nunes ED, Costa PS. Os cursos de saúde coletiva no Brasil mestrado e doutorado: um estudo sobre as disciplinas básicas. Cien Saude Colet 1997; 2(1/2:72-90.

11. M inayo, M CS. Pós-graduação em saúde coletiva: um projeto em construção. Cien Saude Colet 1997; 2(1/2):53-71.

\section{Colaboradores}

ED Nunes, LE Ferreto, ALO Oliveira, JL Nascimento, NF Barros e M EP Castellanos participaram da pesquisa das informações e classificação das disciplinas, sendo que a redação final coube a ED Nunes.
12. Barros JDA. História Econômica:considerações considerações sobre um campo disciplinar. Rev. Economia Política e História Econômica 2008; 1:128-132.

13. Prado M M, Pimentel VL. O campo disciplinar do patrimônio. [site da Internet] [acessado 2009 set 14]. Disponível em: http://www.abracor.com.br/.../ O\%20campo\%20disciplinar\%20do\%20patrimonio

14. Cabral CG. Mapeando e refletindo a construção do campo disciplinar "Estudos feministas da ciência e da tecnologia" no Brasil nos percursos do Fazendo Gênero". [site da Internet] [acessado 2009 set 14]. Disponível em: http://www.fazendogenero8.ufsc.br/sts/ .../Carla_Giovana_Cabral_38.pdf

15. Gusmão EM. Prática de ensino: a construção de um campo disciplinar (1967-1974). [site da Internet] [acessado 2009 set 14]. Disponível em: http://www.sbhe. org.br/novo/congressos/cbhe4/coordenadas/eixo02/ Coordenada\%20por\%20Fatima\%20M aria\%20N eves/ Emery\%20M \%20Gusmao\%20-\%20Texto.pdf

Artigo apresentado em $10 / 12 / 2009$

Aprovado em 15/01/2010

Versão final apresentada em 09/03/2010 